\section{A Rare Case of Heterotopic Calcification with a Pleurocutaneous Fistula 31 Years after Chest Wall Irradiation}

\section{Matthew Lamb ${ }^{1}$, John R Duttenhaver ${ }^{2}$, Daniel Most ${ }^{3}$ and Yaron Perry ${ }^{4 *}$}

${ }^{1}$ Mercer University Medical School - Savannah Campus, USA

${ }^{2}$ Chairman, Radiation Oncology, Memorial Health Medical Center - Savannah, GA, USA

${ }^{3}$ Most Plastic and Reconstructive Surgery, P.C., Memorial Health Medical Center - Savannah, GA, USA

${ }^{4}$ Director Minimally Invasive Thoracic Surgery, Anderson Cancer Institute and Memorial Health Medical Center - Savannah, GA, USA

\begin{abstract}
The rare events of Heterotopic Calcification $(\mathrm{HC})$ as a late complication of radiotherapy have been reported in the past. We report a case of a pleurocutaneous fistula with an open sinus tract in the right chest wall. The fistula formed 31 years ago after radical mastectomy and external beam radiation for stage 2 breast cancer. The HC and fistula were treated with lung and chest wall resection followed by musculocutaneous pedicle flap reconstruction. The patient recovered well with no respiratory sequella.
\end{abstract}

\section{Case Report}

A 65-year-old Caucasian female underwent a modified radical mastectomy for stage 2 infiltrating ductal carcinoma of her right breast in 1982. Original diagnosis March 1989 TNM stage T2 N3a M0 $(3.5 \mathrm{~cm}$ poorly differentiated primary and 20 of $27 \mathrm{LN}+$ with largest node $2.2 \mathrm{~cm}$ with Extracapsular extension). She was given post-operative external beam irradiation: three fields $1 \& 2$ reverse hockey stick to supraclavicular/axilla/lateral chest wall with $6 \mathrm{mv}$ xray from AP and 15mv X-ray from PA. Enface Chest wall 6mev electrons @80\% no bolus.
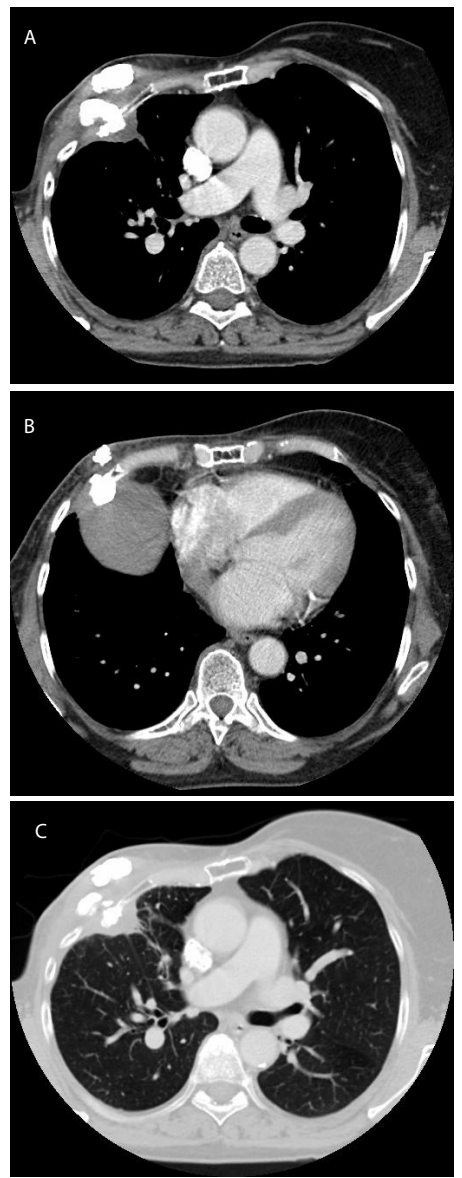

Figure 1: A. calcifications involving the chest wall and the pleural space B. Calcifications involving the diaphragm.

C. Bronchu-cutaneous fistula documented in lung windows.
Dose $=50 \mathrm{~Gy}$ in 25 fractions of $2 \mathrm{~Gy}$ per fraction. $1 \mathrm{~cm}$ match move at $26 \mathrm{~Gy}$ between photon reverse hockey and enface $6 \mathrm{mev}$ electron field. Final mastectomy electron scar boost of $10 \mathrm{~Gy}$ in 5 fractions of $2 \mathrm{~Gy} /$ fx@80\%.

The appearance of the calcifications detected on chest X-rays from 2004 thought to be related to three rib fractures in the 3rd 4th and 5th anterior ribs in the $6 \mathrm{mev}$ electron port in an area that got 50Gy - 60Gy and was otherwise asymptomatic. In April of 2012, the patient presented with significant calcification over her right chest wall exacerbated by bloody drainage from a sinus tract that involved the lung tissue. On physical exam, the patient was afebrile without any signs of systemic infection with audible air leak from the sinus opening on her chest wall. A CT of her chest showed multiple pulmonary nodules and increased calcification along her anterior right chest wall with adhesion of her diaphragm. The scan also confirmed the presence of a sinus tract to her right lung. A PET/CT was done and did not show any evidence of metastatic disease, although it was found that the sinus tract led into her right upper and right middle lobe. The decision was made to perform a chest wall resection and reconstruction to eliminate the fistula (Figure 1).

Video Assisted Thoracoscopy was performed under general anesthesia: the middle and upper lobes were both plastered anteriorly to the chest wall creating a sinus tract into the chest wall calcifications. An Endo-GIA stapler (Covidien) was used to divide and remove the attached peripheral segments of the lobes, leaving the central portions of the upper and middle lobes intact. The diaphragm was adhered to the chest wall; a $5 \mathrm{~cm} \times 4 \mathrm{~cm}$ portion of it was resected as well. In order to divide the anterior chest wall an external open approach used, leaving a $12 \mathrm{~cm} \times 5 \mathrm{~cm}$ elliptical shape defect (Figures 2 and 3). The skin and all underlying tissue with the calcification, including sections of the $4^{\text {th }}-10^{\text {th }}$ ribs, were removed in the segment of the chest wall. The diaphragm was repaired with interrupted \#1 Prolene mattress with

*Corresponding author: Yaron Perry MD, FACS - Director Minimally Invasive Thoracic Surgery, Anderson Cancer Institute and Memorial Health Medical Center-Savannah, GA, USA, E-mail: perryya1@memorialhealth.com

Received April 15, 2013; Accepted May 27, 2013; Published May 29, 2013

Citation: Lamb M, Duttenhaver JR, Most D, Perry Y (2013) A Rare Case of Heterotopic Calcification with a Pleurocutaneous Fistula 31 Years after Chest Wall Irradiation. J Pulmon Resp Med S14: 004. doi:10.4172/2161-105X.S14-004

Copyright: $\odot 2013$ Lamb M, et al. This is an open-access article distributed under the terms of the Creative Commons Attribution License, which permits unrestricted use, distribution, and reproduction in any medium, provided the original author and source are credited. 
pledgets (Figures 4 and 5) and then as an onlay, an AlloDerm patch of $3 \mathrm{~cm} \times 6 \mathrm{~cm}$ was put as an over-patch on the diaphragm. A $30 \times 20 \mathrm{~cm}$ Parietex Covidien mesh was tailored to the defect on the chest wall and this was used to completely cover the defect. Methylmethacrylate was molded over the mesh to give stiffness to the reconstructed chest wall. A latissimus muscle flap and skin grafts were created over the defect (Figure 5). Chest tubes were then secured to the chest via suture. All the thoracoscopic incisions were closed using 3-0 Vicryl and 4-0 Momcryl for the skin.

The Patient was discharged on post op day 5 without any post-op complications. On a 2 week, follow up there was a good uptake of the graft without any signs of infection and appropriate expansion of her right lung on chest-X-ray.

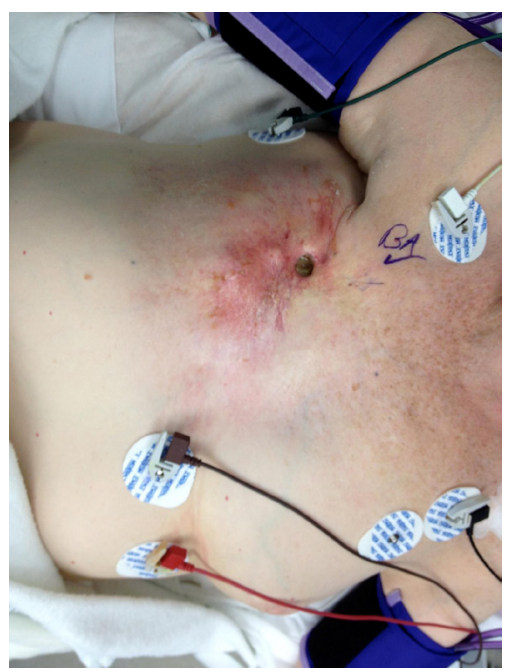

Figure 2: The opening in the skin of the broncho-cutaneous fistula in the mastectomy scar.

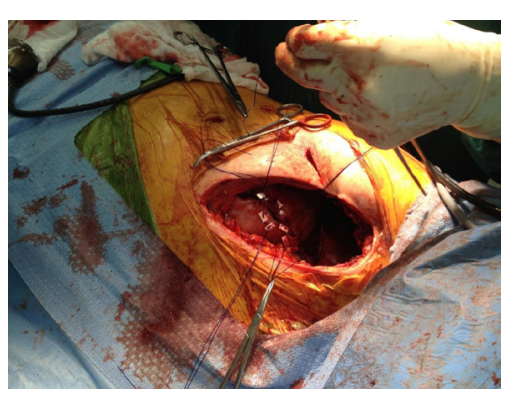

Figure 3: The resected chest wall and the diaphragmatic defect - shown with stay sutures.

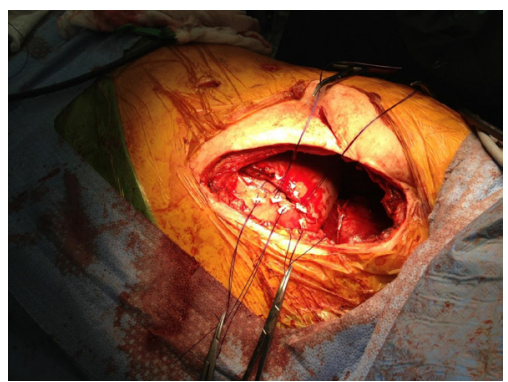

Figure 4: The resected chest wall and the repair of the diaphragmatic defect.

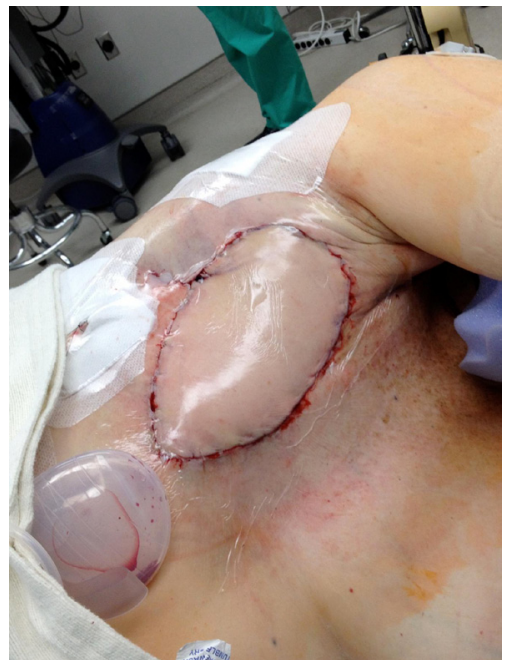

Figure 5: The total reconstruction with lattissimus muscle - musculucutaneous pedicle flap.

Her Pathology from the chest wall resection documented: fibrosis, mixed inflammation and dystrophic calcification with osseous metaplasia with no sign of recurrence or malignancy - correlating with the diagnosis of heterotropic calcification.

\section{Discussion}

Heterotopic Calcification (HC) of tissue after radiation therapy is a rare complication that has been documented in the past [1,2]. HC can occur in tissue after resection of known previous malignancy, as well as in normal tissue irradiated because of previous cancer removal. Usually $\mathrm{HC}$ develops 10 to 20 years after the radiation treatment. Most of the calcification are asymptomatic and unless cosmetically deforming it is left untreated. Rarely when calcified tissue left untreated, breakdown can occur and result in fistula formations. A few cases were described however, when the finding of a fistula is added to the calcification [3] To date, no other report has been documented where a fistula from post irradiation therapy reaches lung tissue, making this case unique. This patient had a clear fistula to both the right pleural space and to lung parenchyma, which probably have formed at a site of the overlap of irradiation treatment. Other factors to consider are her original radiation dosage, her age, and the extended amount of time since her treatment. Earlier treatment of this patient may have prevented the pleurocutaneous fistula to develop. Nowadays more focal and lower doses of external beam radiation will hopefully make such pathology a rare occasion. Nevertheless, good survival rate of breast cancer patients treated 30-15 years ago, which now reach their 60's-80's, makes this case important since we may see more cases like this in a next $10-15$ years. We would like to make the medical community, especially pulmonologists and primary care providers aware that patients treated with high dose external beam radiation 20 or more years ago and presented with initial calcifications has to be considered at risk for fistula development, if not treated immediately and with close follow-up.

\section{References}

1. Carl UM, Hartmann KA (2002) Heterotopic calcification as a late radiation effect: report of 15 cases. Br J Radiol 75: 460-463.

2. Amin R, Hamilton-Wood C, Silver D (2002) Subcutaneous calcification following chest wall and breast irradiation: a late complication. Br J Radiol 75: 279-82.

3. Rudolph R, Griffeth WD (2012) Extensive Breast Calcification in a Patient with History of Partial Mastectomy and Postoperative Radiation Therapy. American Surgeon 78: 139-141. 